\title{
Vulnerability analysis of large concrete dams using the continuum strong discontinuity approach and neural networks
}

\author{
Manolis Papadrakakis ${ }^{\text {a,* }}$, Vissarion Papadopoulos ${ }^{\text {a }}$, Nikos D. Lagaros ${ }^{\text {a }}$, \\ Javier Oliver $^{\mathrm{b}}$, Alfredo E. Huespe ${ }^{\mathrm{c}}$, Pablo Sánchez ${ }^{\mathrm{c}}$ \\ ${ }^{a}$ Institute of Structural Analysis and Seismic Research, National Technical University of Athens, Athens 15780, Greece \\ b Technical University of Catalonia (UPC), Campus Nord UPC, Edifici C-1, ClJordi Girona 1-3, 08034 Barcelona, Spain \\ c CIMEC-INTEC-UNL-CONICET, Guemes 3450, S3000GLN, Santa Fe, Argentina
}

Received 8 February 2006; accepted 21 November 2006

Available online 25 January 2007

\begin{abstract}
Probabilistic analysis is an emerging field of structural engineering which is very significant in structures of great importance like dams, nuclear reactors etc. In this work a Neural Networks (NN) based Monte Carlo Simulation (MCS) procedure is proposed for the vulnerability analysis of large concrete dams, in conjunction with a non-linear finite element analysis for the prediction of the bearing capacity of the Dam using the Continuum Strong Discontinuity Approach. The use of NN was motivated by the approximate concepts inherent in vulnerability analysis and the time consuming repeated analyses required for MCS. The Rprop algorithm is implemented for training the NN utilizing available information generated from selected non-linear analyses. The trained $\mathrm{NN}$ is then used in the context of a MCS procedure to compute the peak load of the structure due to different sets of basic random variables leading to close prediction of the probability of failure. This way it is made possible to obtain rigorous estimates of the probability of failure and the fragility curves for the Scalere (Italy) dam for various predefined damage levels and various flood scenarios. The uncertain properties (modeled as random variables) considered, for both test examples, are the Young's modulus, the Poisson's ratio, the tensile strength and the specific fracture energy of the concrete.
\end{abstract}

(c) 2006 Published by Elsevier Ltd.

Keywords: Reliability analysis; Fragility curves; Vulnerability; Monte Carlo Simulation; Soft computing; Neural Networks; Continuum Strong Discontinuity Approach

\section{Introduction}

The theory and methods of structural vulnerability have been developed significantly during the last twenty years and have been documented in an increasing number of publications. These advancements in

\footnotetext{
${ }^{*}$ Corresponding author.

E-mail address: mpapadra@central.ntua.gr (M. Papadrakakis).
} 
structural reliability theory and the attainment of more accurate quantification of the uncertainties associated with structural loads and resistances have stimulated the interest in the probabilistic treatment of structures. The vulnerability of a structure, or its probability of failure under various loading scenarios, is an important factor in the design, construction, monitoring and maintenance procedures of dams since it investigates the probability of the structure to successfully complete its design requirements. Therefore, vulnerability analysis is at the heart of risk analysis methodologies that have been developed for very important structures like dams and subsidies essentially the decision making procedures, leading to safety measures that the owners and the dam engineers have to take into account due to the aforementioned uncertainties. Although from a theoretical point of view the field has reached a stage where the developed methodologies are becoming widespread, from a computational point of view serious obstacles have been encountered in practical implementations.

First and second order reliability methods that have been developed to estimate structural reliability [1-3] lead to elegant formulations requiring prior knowledge of only the means and variances of the component random variables and the definition of a differentiable failure function. For small-scale problems these types of methods prove to be very efficient, but for large-scale problems and/or large number of random variables Monte Carlo Simulation (MCS) methods seem to be superior. In fact, simulation methods are the only methods available to treat practical reliability problems in complex structures where a complete non-linear threedimensional modelling is required (for example capturing the failure mode mechanisms in dams where the arch-effect, or vault-effect, plays a major role in the structural response [4]). Furthermore, in this type of structures is not possible to establish a priori the failure surfaces determining the structural collapse.

The basic MCS is simple to use, but for typical structural reliability problems the computational effort involved becomes excessive due to the enormous sample size and consequently the CPU time required for each
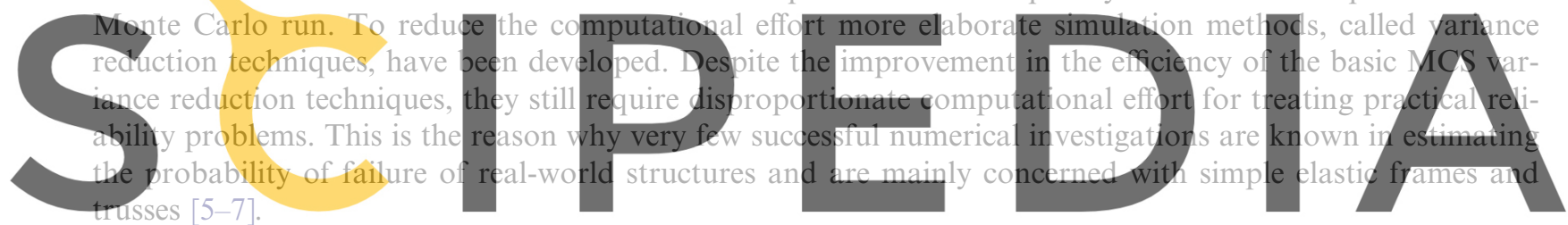

In the present paper a Neural Networks (NN) based Monte Carlo Simulation (MCS) procedure is pro-

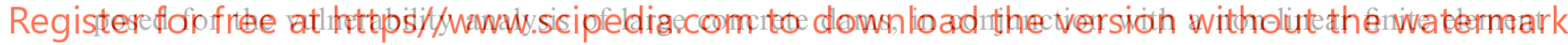
analysis for the prediction of the bearing capacity of the dam using the Continuum Strong Discontinuity Approach (CSDA) [4,8]. The use of artificial intelligence techniques, such as Neural Networks (NN), in structural reliability analysis has been reported in a number of publications [9-11], in which the efficiency as well as the accuracy of this methodology for obtaining close predictions of the probability of failure in complex structural systems is demonstrated. The principal advantage of a properly trained NN is that it requires a trivial computational effort to produce an acceptable approximate solution. The Rprop algorithm is implemented for training the NN utilizing available information generated from selected non-linear analyses. The trained NN is then used in the context of a MCS procedure to compute the peak load of the dam due to different sets of basic random variables leading to close prediction of its probability of failure.

Once a NN is trained to produce acceptable estimates of the peak loads of the dam, rigorous calculations of fragility curves can be subsequently obtained by performing a number of reliability analyses associated with various predefined damage levels of the dam and various flood scenarios. The proposed combined NN-CSDA methodology is first demonstrated through a simple "academic" example involving a concrete beam test subjected to a loading system placed in four points. From this initial demonstration, the capacity of the CSDA to model and predict material failure is assessed through comparisons with corresponding experimental results, while the ability of the NN to predict close estimates of the peak loads with and consequently of the probability of failure is validated. The aforementioned calculations are then repeated for the vulnerability analysis of Scalere (Italy) dam demonstrating the efficiency as well as the applicability of the proposed methodology in large and complex structural systems. The uncertain properties (modeled as random variables) considered for this example are the Young's modulus, the Poisson's ratio, the tensile strength and the specific fracture energy of the concrete. 


\section{Continuum Strong Discontinuity Approach to material failure}

\subsection{Motivation}

Phenomenological modeling of material failure, in structural mechanics, aims at capturing the formation of those macroscopically observable discontinuities in the displacement field which, depending on the context, are termed, cracks, fractures, slip lines, etc. They are identified as the locus of those material points where loss, and eventual exhaustion, of the material capacity to carry stresses (material failure) takes place, and, as they spread through the structure, are responsible for the progressive reduction of the structural tangent stiffness to zero (identifying the structural failure and the critical load stage) or to negative values (identifying post-critical stages). The mechanical modeling of the onset and progression of displacement discontinuities (technically termed strong-discontinuities) can be approached from a wide range of options which can be classified into two large groups: (1) continuum approaches, based on the use of non-linear continuum (stress-strain) constitutive models, equipped with strain-softening to capture the progressive loss of the strength of the material as strain increases, and (2) discrete approaches (also termed cohesive or non-linear fracture mechanics based approaches) lying on the insertion, at predetermined discontinuity paths, of specific traction-separation laws (also equipped with softening for the same purpose) whereas the bulk of the structure remains in elastic state.

As for numerical procedures, the continuum approaches have been traditionally used in connection with standard finite elements resulting in the so called smeared approaches. They do not require an a priori knowledge of the discontinuity path which is a posteriori identified through those element patches where concentration of intense deformations (strain localization or weak discontinuities) occur. However, there is a strong dependency of the captured discontinuity paths on the mesh orientation (mesh bias dependency); they often
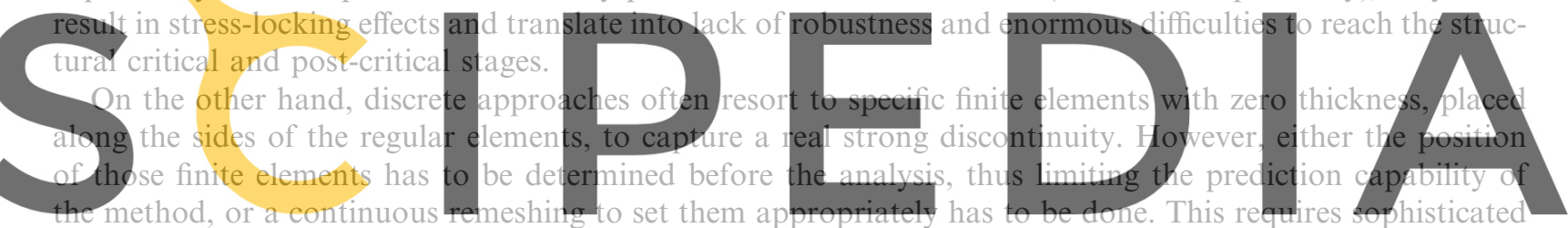

remeshing procedures, leading to unaffordable computational costs when large three-dimensional structures

Registe mforifhe is inttpisiekvawwiscipediaicomp dodownload the version without the watermark

The above considerations justify the very limited capacity of those classical strategies to be applied to vulnerability analysis purposes, based on the Monte Carlo Simulation methods requiring a large number of complex simulations.

In recent years some alternatives have emerged, belonging to the family of the so called strong discontinuity approaches, as an attempt to overcome the problems of the previous approaches. In particular, the continuum strong discontinuity approach (CSDA), which has been adopted here, is equipped with recent and advanced ingredients in order to make it an efficient and robust strategy for solving complex three-dimensional multicrack problems. The fundamental features of CSDA are the following:

- A real displacement discontinuity (crack) is considered in the mathematical description of the displacement field, through the so called strong discontinuity kinematics [12-14], (see Fig. 1). The displacement, u, and strain, $\varepsilon$, fields in a body $\Omega$ experiencing a strong discontinuity across the crack surface, $S$, are then described as

$$
\begin{aligned}
& \mathbf{u}(\mathbf{x})=\overline{\mathbf{u}}(\mathbf{x})+\mathrm{H}_{\mathrm{S}} \llbracket \mathbf{u} \rrbracket(\mathbf{x}) \quad \mathrm{H}_{\mathrm{S}}=\left\{\begin{array}{cc}
1 & \forall \mathbf{x} \in \Omega^{+} \\
0 & \forall \mathbf{x} \in \Omega^{-}
\end{array}\right. \\
& \boldsymbol{\varepsilon}(\mathbf{x})=\nabla^{S} \mathbf{u}(\mathbf{x})=\underbrace{\overline{\boldsymbol{\varepsilon}}(\mathbf{x})}_{\begin{array}{c}
\text { regular } \\
\text { (bounded) }
\end{array}}+\underbrace{\delta_{\mathrm{S}}(\llbracket \mathbf{u} \rrbracket \otimes \mathbf{n})^{\text {sym }}}_{\begin{array}{c}
\text { singular } \\
\text { (unbounded) }
\end{array}},
\end{aligned}
$$

where $\llbracket \mathbf{u} \rrbracket$ in Eq. (1) is the displacement jump, $H_{S}$ is the step function and $\delta_{S}$ in Eq. (2) is the Dirac's delta function, which is regularized for computational purposes. 

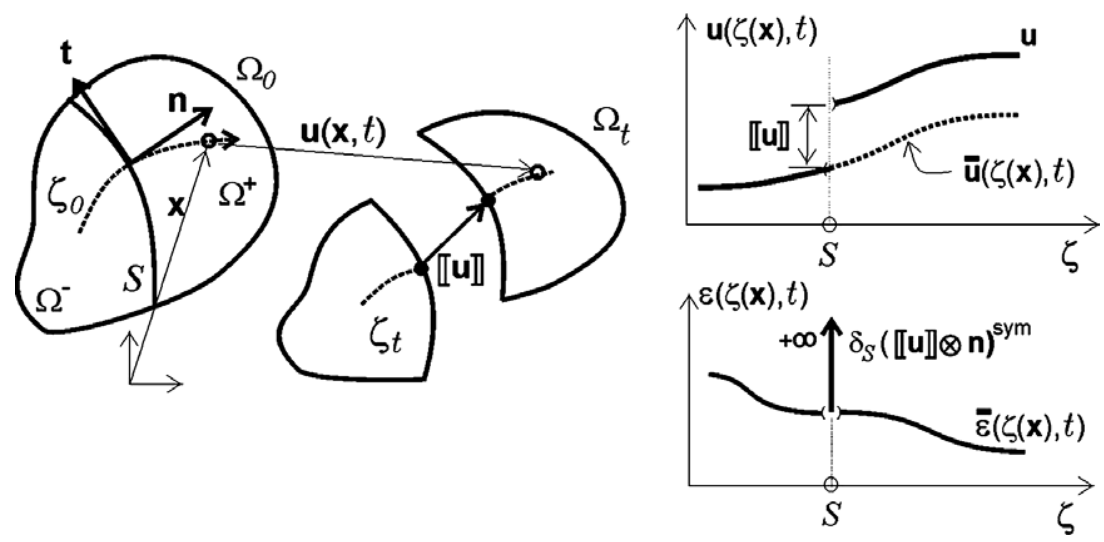

Fig. 1. Strong discontinuity kinematics.

- A unique continuum constitutive model (stress/strain) is used to model both the material behaviour at the bulk and at the discontinuity interface [14]. The model is equipped with strain softening, to reproduce the material de-cohesion as cracking occurs. The softening modulus is regularized to match with the unbounded strains in Eq. (2). Therefore, the complete analysis is performed within a continuum setting. Nevertheless, it can be shown that the selected continuum 3D material model is automatically projected into a 2D discrete model (traction-separation law) at the discontinuity interface as the strong discontinuity kinematics in Eqs. ((1) and (2)) develops. For practical purposes, that discrete model is neither derfved non implemented, but the results

Therefore, both, the volum

tured in a common continu e and surfo he use of finite elements with embedded discontimuities [13] makes

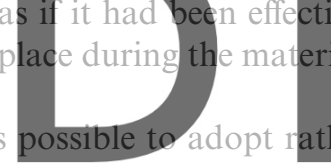
her coarse meshes, without affecting the accuracy of the results (see Fig. 2) and not requiring remeshing procedures. Those ele-

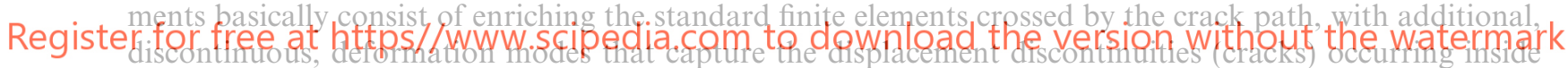

the element. These deformation modes have an elemental support. Therefore, the corresponding enriching degrees of freedom can be condensed at the elemental level, so that the enrichment does not eniarge the size of the system of equations to be solved, and multiple cracks can be captured, even in coarse meshes, at a reduced computational cost.

- The critical conditions for the material failure, and the directions of its propagation, are checked at every step in the whole structure (looking for the singularity of the so called localization tensor [13]). This means that the material cracks are captured without any a priori knowledge of them. Then, a so-called global tracking algorithm [15] is used to determine which elements are capturing the crack path and, therefore, have to be enriched.

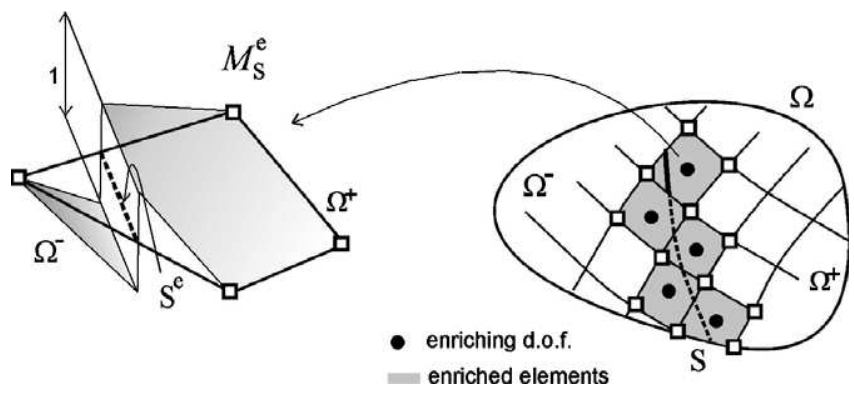

Fig. 2. Finite elements with embedded discontinuities. 
- The (recently introduced) implicit-explicit integration scheme is used to integrate the rate (non-linear) constitutive equations [16]. This ensures positiveness and constancy, during a given time step, of the algorithmic tangent stiffness, and translates into dramatic improvements of the robustness of the linearization procedure and a large reduction (to only one) of the number of iterations per time step. Specific arc-length strategies are implemented to control the error introduced by that implicit-explicit algorithm.

Additional theoretical aspects of the CSDA are described in detail in [12-16].

\subsection{Experimental assessment of the CSDA: the four point bending test}

In order to assess the capacity of the CSDA to model and predict material failure a very well-known experimental benchmark test, widely used for numerical validation in computational failure mechanics, is reproduced here. It corresponds to the four point bending test sketched in Fig. 3a. A notched concrete beam, whose material is characterized by the following parameters: Young's modulus $E=24800 \mathrm{MPa}$, Poisson's ratio $v=0.18$, tensile strength $\sigma_{11}=2.8 \mathrm{MPa}$, fracture energy $G_{f}=100 \mathrm{~N} / \mathrm{m}$, and thickness $156 \mathrm{~mm}$, is supported at two points and subjected to a loading system imposing two forces in points $A$ and $B$. The load-displacement system is controlled until reaching the complete failure of the beam. The experimental setting is shown in Fig. $3 \mathrm{~b}$ and the results have been reported by Arrea and Ingrafea [17].

The experimentally observed failure mode displays a crack crossing the specimen from the tip of the notch toward the load application point $B$, as shown in Fig. 3c and d.

The selected constitutive model to reproduce the material behaviour is a classical isotropic continuum damage model described in [12-14]. The initial behaviour, under increasing strains, is elastic, characterized by the
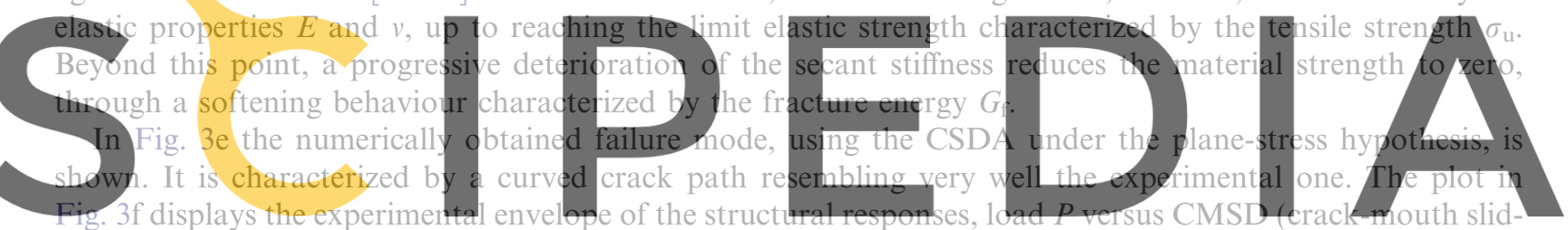

ing displacement) curves, and the numerically obtained results. It can be observed that the numerical struc-

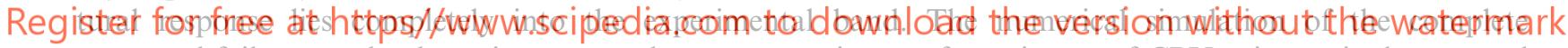
structural failure can be done, in a very robust manner, in very few minutes of CPU using a single personal computer. In addition, the independence of the obtained results on the orientation of the finite element mesh (mesh objectivity) can be shown. This example exemplifies the ability of the selected computational material failure setting (the CSDA) to provide fast and reliable numerical results to be used for the purposes of this work as detailed in subsequent sections.

\section{Structural reliability analysis}

In the design of structural systems, limiting uncertainties and increasing safety is an important issue to be considered. Structural vulnerability, which is defined as the probability that the system meets some specified demands for a specified time period under specified environmental conditions, is used as a probabilistic measure to evaluate the integrity (available risk) of structural systems.

The probability of failure $p_{\mathrm{f}}$ can be determined using a time invariant reliability analysis procedure with the following relationship:

$$
p_{\mathrm{f}}=p[R<S]=\int_{-\infty}^{\infty} F_{R}(t) f_{S}(t) \mathrm{d} t=1-\int_{-\infty}^{\infty} F_{S}(t) f_{R}(t) \mathrm{d} t,
$$

where $R$ denotes the structure's bearing capacity and $S$ the external loads. The randomness of $R$ and $S$ can be described by known probability density functions $f_{R}(t)$ and $f_{S}(t)$, with $F_{R}(t)=p[R<t], F_{S}(t)=p[S<t]$ being the cumulative probability density functions of $R$ and $S$, respectively.

Most often a limit state function is defined as $G(R, S)=S-R$ and the probability of structural failure is given by 
a

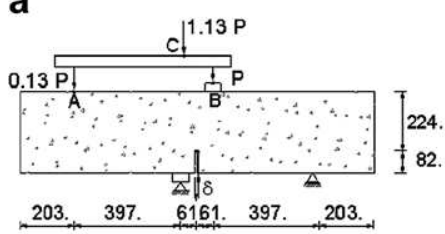

b

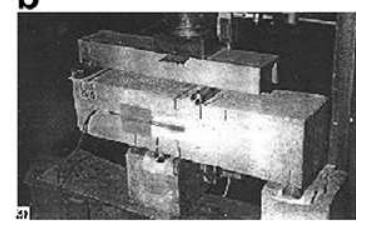

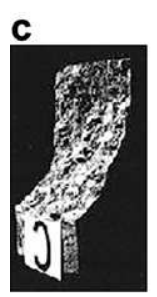

d
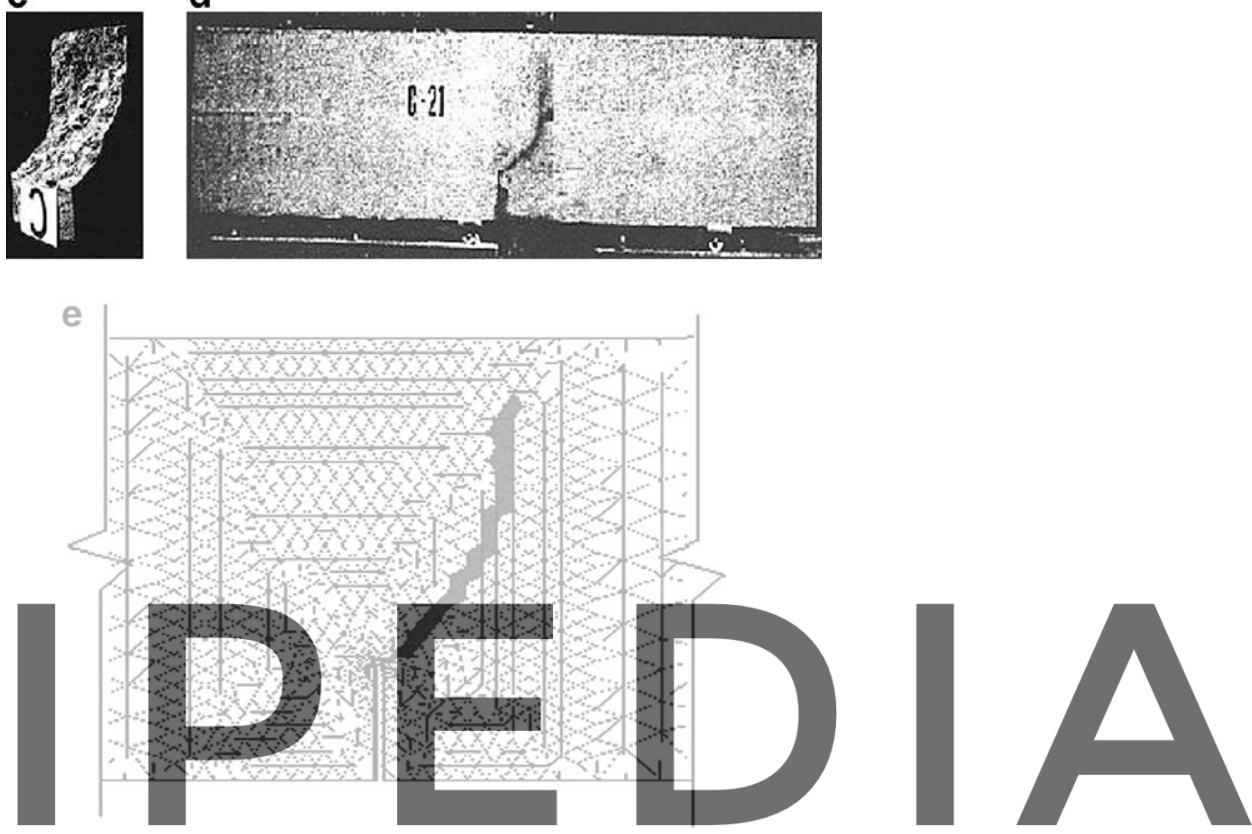

Register for free at https//WwW.scipediancomettodownload the version without the watermark

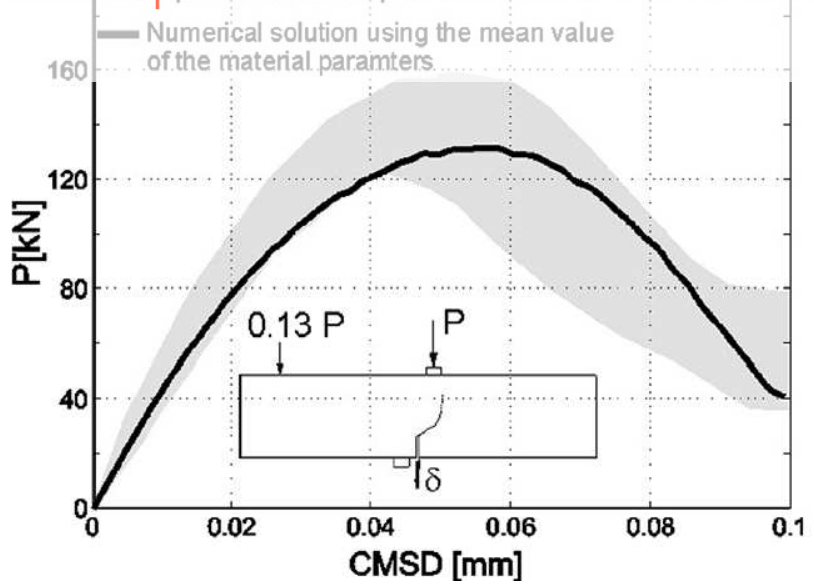

Fig. 3. Four-point bending test. (a) Geometrical description. (b) Experimental setup. (c) Crack surface after the experiment. (d) Experimental crack. (e) Numerically obtained crack path. (f) Numerical and experimental structural responses.

$$
p_{\mathrm{f}}=p[G(R, S) \geqslant 0]=\int_{G \geqslant 0} f_{R}(R) f_{S}(S) \mathrm{d} R \mathrm{~d} S .
$$

It is practically impossible to evaluate $p_{\mathrm{f}}$ analytically for complex and/or large-scale structures, especially in the case of the vulnerability analysis of large concrete dams considered in the present study. In such cases the integral of Eq. (4) can be calculated only approximately using either simulation methods, such as the Monte 
Carlo Simulation (MCS), or approximation methods like the first order reliability method (FORM) and the second order reliability method (SORM), or response surface methods (RSM) [1-3,18]. Despite its high computational cost, MCS is considered as a reliable method and is commonly used for the evaluation of the probability of failure in computational mechanics, either for comparison with other methods or as a standalone reliability analysis tool.

In reliability analysis the MCS method is often employed when an analytical solution is not attainable and the failure domain can not be expressed or approximated by an analytical form. This is mainly the case in problems of complex nature with a large number of basic variables where all other reliability analysis methods are not applicable. Expressing the limit state function as $G(x)<0$, where $x=\left(x_{1}, x_{2}, \ldots x_{M}\right)$ is the vector of the random variables, Eq. (4) can be written as

$$
p_{\mathrm{f}}=\int_{G(x) \geqslant 0} f_{x}(x) \mathrm{d} x
$$

where $f_{x}(x)$ denotes the joint probability of failure for all random variables. Since MCS is based on the theory of large numbers $\left(N_{\infty}\right)$ an unbiased estimator of the probability of failure is given by

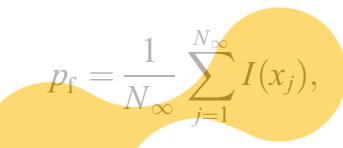

in which $I\left(x_{j}\right)$ is a failure indicator for each simulation defined as

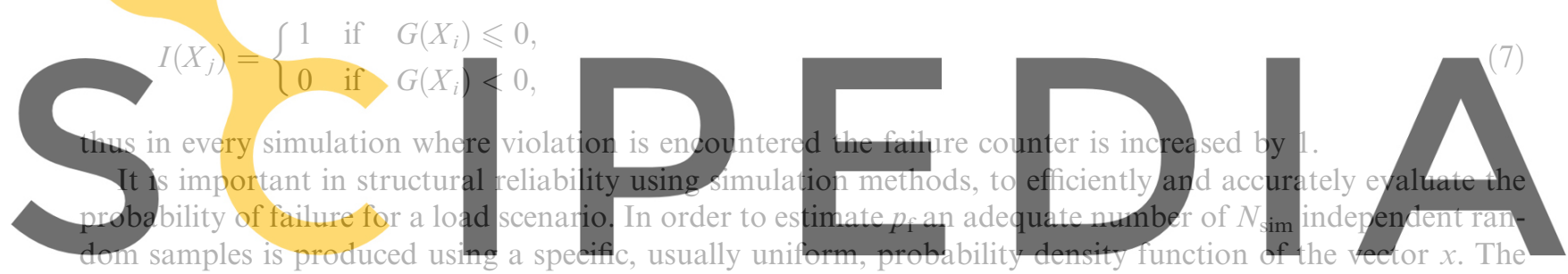

value of the failure function is computed for each random sample $x_{j}$ and the Monte Carlo estimation of $p_{\mathrm{f}}$ is

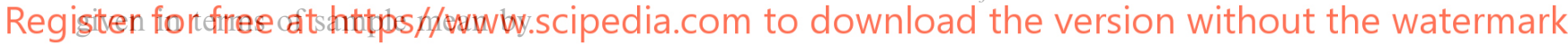

$$
p_{\mathrm{f}} \cong \frac{N_{\mathrm{H}}}{N_{\text {sim }}},
$$

where $N_{\mathrm{H}}$ is the number of successful simulations.

\section{Multi-layer perceptrons}

A multi-layer perceptron is a feed-forward neural network, consisting of a number of units (neurons) linked together that attempts to create a desired relation in an input/output set of learning patterns. A learning algorithm tries to determine a set of parameters called weights, in order to achieve the right response for each input vector applied to the network. The numerical minimization algorithms used for the training generate a sequence of weight matrices through an iterative procedure. To apply an algorithmic operator $\mathrm{A}$ we need a starting weight matrix $w^{(0)}$, while the iteration formula can be written as follows:

$$
w^{(t+1)}=\mathrm{A}\left(w^{(t)}\right)=w^{(t)}+\Delta w^{(t)} .
$$

All numerical methods applied are based on the above formula. The changing part of the algorithm $\Delta w^{(t)}$ is further decomposed into two parts as

$$
\Delta w^{(t)}=a_{t} d^{(t)},
$$

where $d^{(t)}$ is a desired search direction of the move and $a_{t}$ the step size in that direction. 
The training methods can be divided into two categories. Algorithms that use global knowledge of the state of the entire network, such as the direction of the overall weight update vector, which are referred to as global techniques. In contrast, local adaptation strategies are based on weight specific information only such as the temporal behaviour of the partial derivative of this weight. The local approach is more closely related to the neural network concept of distributed processing in which computations can be made independent to each other. Furthermore, it appears that for many applications, local strategies achieve faster and reliable prediction than global techniques despite the fact that they use less information [19].

\subsection{Global adaptive techniques}

The algorithms most frequently used in the NN training are the steepest descent, the conjugate gradient and the Newton's methods with the following direction vectors:

Steepest descent method: $d^{(t)}=-\nabla \mathrm{E}\left(w^{(t)}\right)$.

Conjugate gradient method: $d^{(t)}=-\nabla \mathrm{E}\left(w^{(t)}\right)+\beta_{t-1} d^{(t-1)}$ where $\beta_{t}$ is defined:

$\beta_{t-1}=\nabla \mathrm{E}_{t} \cdot \nabla \mathrm{E}_{t} / \nabla \mathrm{E}_{t-1} \cdot \nabla \mathrm{E}_{t-1}$ Fletcher-Reeves.

Newton's method: $d^{(t)}=-\left[H\left(w^{(t)}\right)\right]^{-1} \nabla \mathrm{E}\left(w^{(t)}\right)$.

The convergence properties of optimization algorithms for differentiable functions depend on properties of the first and/or second derivatives of the function to be optimized. When optimization algorithms converge slowly for neural network problems, this suggests that the corresponding derivative matrices are numerically ill-conditioned. It has been shown that these algorithms converge slowly when rank-deficiencies appear in the Jacobian matrix of a neura

$(2$

(1)

Local adaptive techniques

To improve the performance of weight updating, two completely

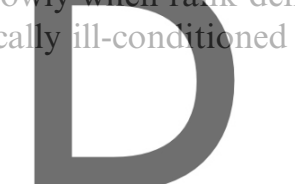

namely Quickprop [21] and Rprop [22].

Register for free at https//WwW.scipedia.com to download the version without the watermark

\subsubsection{The Quickprop method}

This method is based on a heuristic learning algorithm for a multi-layer perceptron, developed by Fahiman [21], which is partially based on the Newton's method. Quickprop is one of most frequently used adaptive learning paradigms. The weight updates are based on estimates of the position of the minimum for each weight, obtained by solving the following equation for the two following partial derivatives:

$$
\frac{\partial \mathrm{E}_{t-1}}{\partial w_{i j}} \text { and } \frac{\partial \mathrm{E}_{t}}{\partial w_{i j}}
$$

and the weight update is implemented as follows:

$$
\Delta w_{i j}^{(t)}=\frac{\frac{\partial \mathrm{E}_{t}}{\partial w_{i j}}}{\frac{\partial \mathrm{E}_{t-1}}{\partial w_{i j}}-\frac{\partial \mathrm{E}_{t}}{\partial w_{i j}}} \Delta w_{i j}^{(t-1)} .
$$

The learning time can be remarkably improved compared to the global adaptive techniques.

\subsubsection{The Rprop method}

Another heuristic learning algorithm with locally adaptive learning rates based on an adaptive version of the Manhattan-learning rule and developed by Riedmiller and Braun [22] is the Resilient backpropagation abbreviated as Rprop. The weight updates can be written

$$
\Delta w_{i j}^{(t)}=-\eta_{i j}^{(t)} \operatorname{sgn}\left(\frac{\partial \mathrm{E}_{t}}{\partial w_{i j}}\right)
$$


where

$$
\eta_{i j}^{(t)}= \begin{cases}\min \left(\alpha \cdot \eta_{i j}^{(t-1)}, \eta_{\max }\right), & \text { if } \quad \frac{\partial \mathrm{E}_{t}}{\partial w_{i j}} \cdot \frac{\partial \mathrm{E}_{t-1}}{\partial w_{i j}}>0, \\ \max \left(b \cdot \eta_{i j}^{(t-1)}, \eta_{\min }\right), & \text { if } \frac{\partial \mathrm{E}_{t}}{\partial w_{i j}} \cdot \frac{\partial \mathrm{E}_{t-1}}{\partial w_{i j}}<0, \\ \eta_{i j}^{(t-1)}, & \text { otherwise, }\end{cases}
$$

where $\alpha=1.2, b=0.5, \eta_{\max }=50$ and $\eta_{\min }=0.1$ [23]. The learning rates are bounded by upper and lower limits in order to avoid oscillations and arithmetic underflow. It is interesting to note that, in contrast to other algorithms, Rprop employs information about the sign and not the magnitude of the gradient components. In this work the Rprop learning algorithm is used since it has been proved by the authors the most efficient one in terms of generalization and rapid convergence of the training procedure [24].

\section{3. $N N$ based $M C$ simulation}

Vulnerability analysis of large concrete dams using Monte Carlo Simulation is a highly intensive computational problem which makes conventional approaches incapable of treating large scale problems even in today's powerful computers. In the present study the use of NN was motivated by the approximation concepts inherent in vulnerability analysis. The idea here is to train a NN to provide computationally inexpensive estimates of analysis outputs required for the reliability analysis problem. The major advantage of a trained NN over the conventional process, under the provision that the predicted results fall within acceptable tolerances, is that results can be produced in a few clock cycles, representing orders of magnitude less computational effort
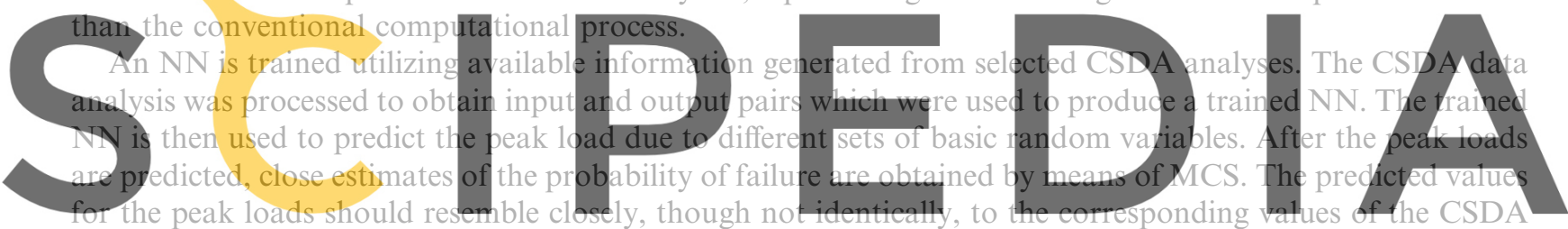

analyses which are considered "exact". It appears that the use of a properly selected and trained NN can elim-

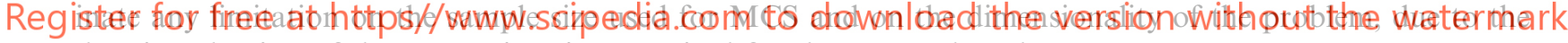
drastic reduction of the computing time required for the repeated analyses.

As it was mentioned, earlier the main objective of this study is to investigate the ability of the NN to predict the peak load and incorporate the NN in the framework of reliability analysis. This objective comprises the following tasks: (i) select the proper training/testing set, (ii) find the suitable neural network architecture and (iii) train the neural network. The appropriate selection of input-output training data is one of the most important parts in NN training. Although the number of training patterns may not be the only concern, the distribution of samples is of greater importance. The number of conventional CSDA analysis calculations performed in this study ranges between 50 and 200 samples, while some of them are used to test the accuracy of the NN predictions with respect to the "exact" results obtained from the CSDA. The selection of the training set is based on the requirement that the full range of possible results should be represented in the training procedure. In order to fulfil the requirement that the full range of possible results should be represented, the training set is generated randomly using the Latin Hypercube Sampling within the bounds: mean value $\pm 6 \sigma$, where the percent variation is 99.9999998 , since in both test examples considered the random variables follow the normal distribution.

There are typically two types of networks, namely fully and patterned connected networks. In this work a fully connected network is used. In a fully connected network, each node in a layer is connected to all the nodes of the previous and the next layer. The number of nodes to be used in the hidden layers is not known in advance, for this reason the learning process initiates with a relatively small number of hidden nodes (10 in this study) and gradually increase the number of hidden nodes and next, until achieving the desired convergence. The NN configuration achieving the desired convergence, for both test examples, has one hidden layer with 20 hidden nodes. Tests performed for more than one hidden layer showed no significant improvement in the obtained results $[9,20]$. 
After the selection of the suitable $\mathrm{NN}$ architecture and the training procedure, the network is then used to provide predictions of the peak load corresponding to different values of the input random variables. The results are then processed by means of MCS to calculate the probability of failure $p_{\mathrm{f}}$. Similarly for the vulnerability analysis of concrete dams using the MCS, the computed peak loads are compared to the corresponding external loading leading to the computation of the probability of structural failure according to Eq. (6). The considered external loads correspond to various flood scenarios and include a constant selfweight and an increasing fictitious density of the water (and the corresponding hydrostatic pressure). It must be mentioned here that by approximating the "exact" solution with a NN prediction of the peak load, the accuracy of the predicted $p_{\mathrm{f}}$ depends not only on the accuracy of the NN prediction of the peak load but also on the sensitivity of $p_{\mathrm{f}}$ with regard to a slightly modified, due to the NN approximation, sample space of resistances [9].

\section{Numerical results}

Two test examples have been considered to illustrate the efficiency of the proposed NN based methodology, one academic and one real world test example. In both test examples the probability of failure is estimated using the basic MCS. Elastic modulus, Poisson's ratio, tensile strength and fracture energy are considered to be random variables in both test examples, following the normal distribution. The NN software used in this study is based on the back propagation algorithm developed by the authors [20].

5.1. Assessment of the combined NN-CSDA methodology: the four point bending test

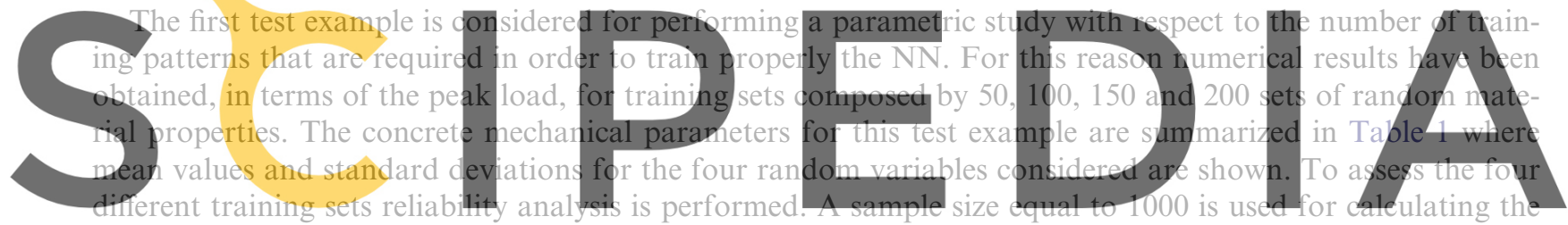

probability of failure. The corresponding external loading, leading to the computation of the probability of

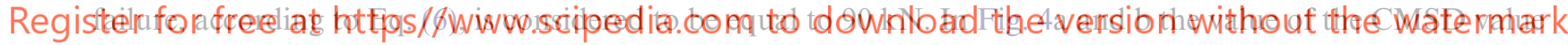
and the peak load for each of the 1000 samples is depicted, while in Fig. 5 the load versus the CMSD curve for all the samples is shown.

The four different training sets are first compared with respect to their prediction ability in a selected set of 10 patterns randomly selected from the 1000 samples mentioned above. The capability of the four training sets to predict the peak load is shown in Table 2. As it can be observed the peak load predicted based on the NN50 training set is the worst one while the NN100, NN150 and NN200 perform equally well with respect to the CDSA prediction. As it was mentioned earlier, once an acceptable trained $\mathrm{NN}$ is obtained in predicting the peak loads, the probability of failure for each test case is estimated by means of the NN-based Monte Carlo Simulation. The results of this calculation are provided in Table 3. The probability of failure for the case of the exact calculations $p_{\mathrm{f} \_\mathrm{CDSA}}=1.00 \%$ while for the case of the neural networks $p_{\mathrm{f} \_N N 50}=0.60 \%$, $p_{\mathrm{f} \_\mathrm{NN} 100}=0.90 \%, p_{\mathrm{f} \_\mathrm{NN} 150}=1.00 \%$ and $p_{\mathrm{f} \_\mathrm{NN} 200}=1.00 \%$. According to a previous work of the authors, if the number of samples is increased the NN100 will converge closely to the $p_{\mathrm{f} \_ \text {CDSA }}$ [9].

Table 1

Concrete beam: mechanical parameters

\begin{tabular}{lll}
\hline & Mean value & Standard deviation \\
\hline Concrete beam & & 1087.23 \\
Elastic Modulus (MPa) & 24700 & 0.0108 \\
Poisson's Ratio & 0.18 & 0.448 \\
Tensile strength (MPa) & 2.8 & 4.5 \\
Fracture energy (N/m) & 100 & \\
\hline
\end{tabular}



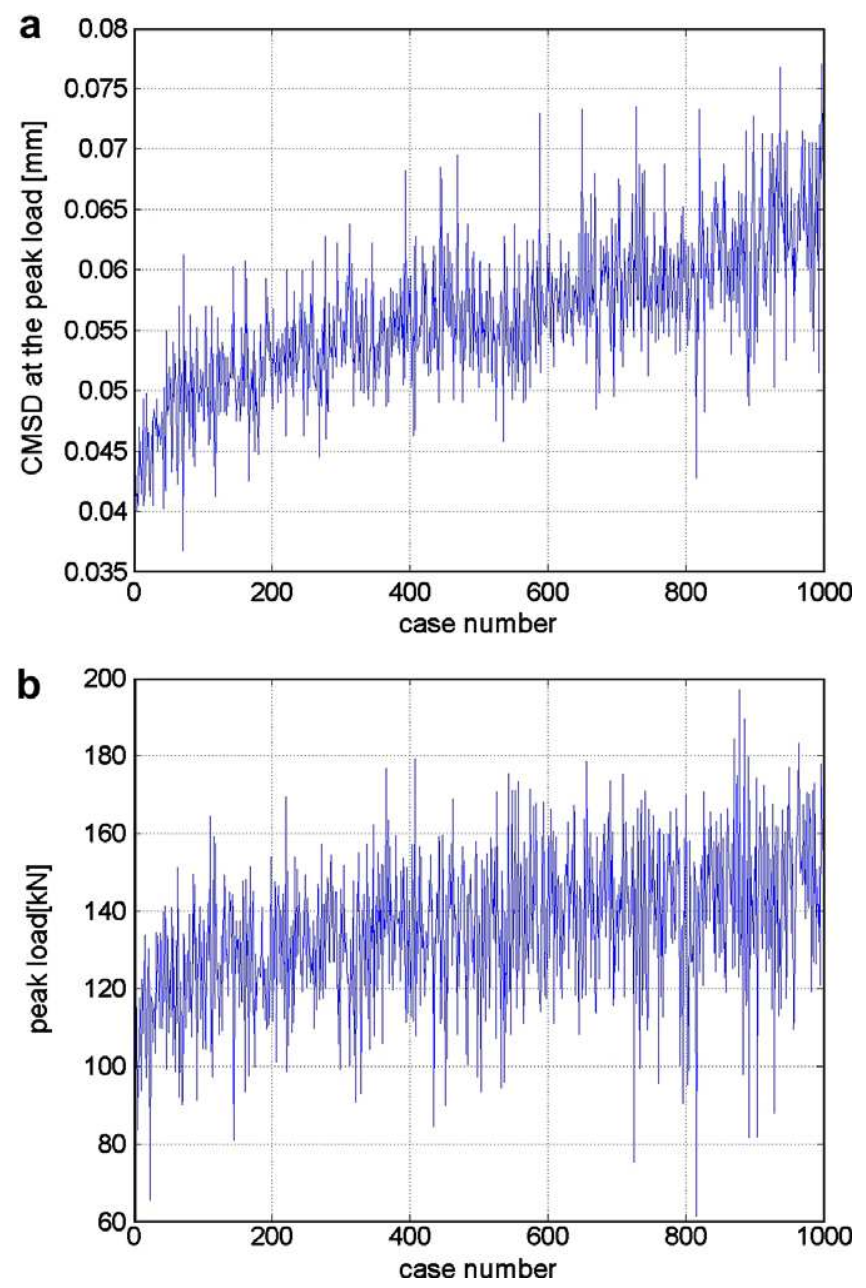

Fig. 4. Four-point bending test. (a) CMSD and (b) peak load for the 1000 samples.

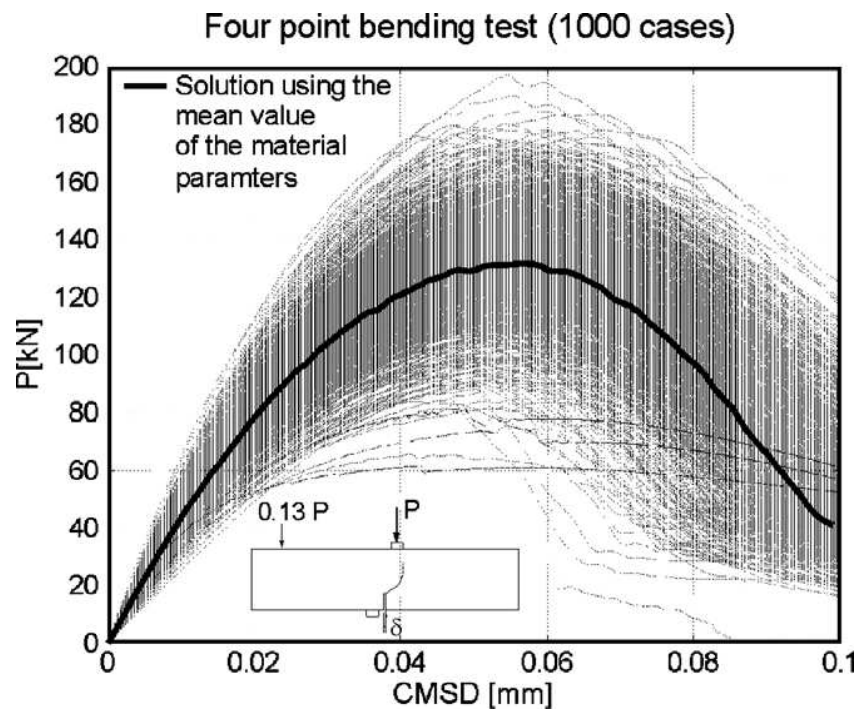

Fig. 5. Four-point bending test. Load versus CMSD curves for the 1000 samples. 
Table 2

Comparison of CDSA ('exact') and NN predictions of the peak load for 10 randomly selected input sets of random variables

\begin{tabular}{|c|c|c|c|c|c|c|c|c|}
\hline \multirow{2}{*}{$\begin{array}{l}\text { Young's } \\
\text { modulus (MPa) }\end{array}$} & \multirow{2}{*}{$\begin{array}{l}\text { Poission's } \\
\text { ratio }\end{array}$} & \multirow{2}{*}{$\begin{array}{l}\text { Tensile strength } \\
(\mathrm{MPa})\end{array}$} & \multirow{2}{*}{$\begin{array}{l}\text { Fracture energy } \\
(\mathrm{N} / \mathrm{m})\end{array}$} & \multicolumn{5}{|c|}{ Peak load $(\mathrm{kN})$} \\
\hline & & & & CSDA & $\begin{array}{l}\text { NN50- } \\
\text { prediction }\end{array}$ & $\begin{array}{l}\text { NN100- } \\
\text { prediction }\end{array}$ & $\begin{array}{l}\text { NN150- } \\
\text { prediction }\end{array}$ & $\begin{array}{l}\text { NN200- } \\
\text { prediction }\end{array}$ \\
\hline 23266 & 0.159 & 2.98 & 56.85 & 117.77 & 114.82 & 115.24 & 115.33 & 115.83 \\
\hline 24603 & 0.177 & 2.89 & 59.54 & 120.32 & 121.48 & 121.33 & 121.38 & 120.69 \\
\hline 23845 & 0.167 & 3.81 & 63.47 & 130.37 & 131.75 & 129.70 & 130.29 & 131.41 \\
\hline 24238 & 0.172 & 2.55 & 64.80 & 117.15 & 117.87 & 118.14 & 118.04 & 117.09 \\
\hline 26451 & 0.203 & 3.28 & 68.11 & 134.34 & 137.55 & 136.97 & 137.54 & 137.41 \\
\hline 24137 & 0.171 & 2.80 & 70.83 & 124.84 & 122.86 & 123.20 & 123.25 & 123.37 \\
\hline 22400 & 0.147 & 2.03 & 72.99 & 104.53 & 107.01 & 107.03 & 107.20 & 106.03 \\
\hline 20852 & 0.126 & 1.56 & 73.38 & 90.09 & 95.07 & 94.62 & 95.35 & 93.74 \\
\hline 20779 & 0.125 & 2.28 & 75.15 & 107.60 & 104.53 & 103.83 & 104.08 & 103.46 \\
\hline 25626 & 0.191 & 3.46 & 77.64 & 141.95 & 140.61 & 139.69 & 140.13 & 140.69 \\
\hline
\end{tabular}

Table 3

Comparison of the probability of failure calculated by the CDSA and the NN based procedure for 1000 simulations (applied load $90 \mathrm{kN}$ )

\begin{tabular}{lllll}
\hline CSDA & NN50-prediction & NN100-prediction & NN150-prediction & NN200-prediction \\
\hline $1.00 \%$ & $0.60 \%$ & $0.90 \%$ & $1.00 \%$ & $1.00 \%$ \\
\hline
\end{tabular}

\subsection{Dam test example}

A large concrete arch dam has been considered as a second test example in order to illustrate the feasibility of the proposed methodology. First, the probability of failure of the Dam is computed for a given water level while at a next step, fragility curves are obtained for various predefined damage levels of the dam and various flood scenarios. The probability of failure for each damage level and each scenario is estimated using the NNbased MCS in conjunction with the CSDA for the prediction of the peak load of the dam. The uncertain properties (modeled as random variables) considered for the Dam are the Young's modulus, the Poisson's ratio, the tensile strength and the specific fracture energy of the concrete. All random variables are assumed to be Gaussian.

\subsubsection{Problem description}

This problem corresponds to the benchmark test presented in the 7th International Benchmark Workshop [25], which here is reproduced using the Continuum Strong Discontinuity Approach. The Scalere dam is an arch dam located in the centre-north of Italy. It was constructed in 1910-1911. The foundation rock consists of stratified Eocene sandstones dipping upstream. The dam has not been provided with contraction joints. The crest elevation is at $830.5 \mathrm{~m}$ a.s.1., maximum height $34.0 \mathrm{~m}$, and crest length $158.0 \mathrm{~m}$. The systematic failure analysis has consisted of fixing all the parameters that are related to the numerical strategy, while changing the four material mechanical properties aforementioned. Therefore, the results will be associated to those physical perturbations.

5.2.1.1. Geometry. The geometry of the concrete dam has been taken from [26]. Only a small part of the foundation has been modelled because the analysis is addressed to the dam structural collapse. Nevertheless, in order to recover the actual structural response, appropriate boundary conditions have been considered. The finite element mesh is displayed in Fig. 6, from different points of view. The discrete model is composed of 13580 tetrahedral elements and 3047 nodes.

5.2.1.2. Boundary conditions. The kinematical restrictions that have been considered are the following: the lateral and bottom surfaces are clamped (surface A, B and C in Fig. 6b), while the other ones are free. 

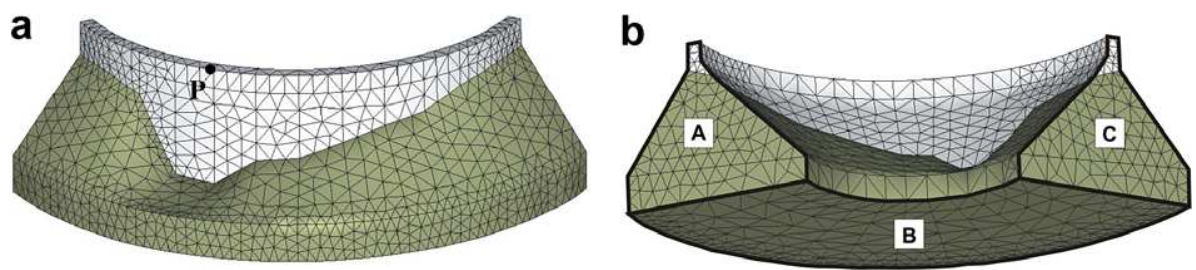

Fig. 6. Dam Geometry and finite element mesh. (a) Upstream view. (b) Inferior view.

The loading action to trace the structural response consists of the so called increasing fictitious water density strategy: the hydrostatic pressure, acting on the up-stream wall of the dam for the full reservoir case, is multiplied by a loading factor (starting from zero) while keeping constant the self-weight of the dam. The maximum attained load factor would be that determining the safety factor for the most unfavourable action (the hydrostatic pressure). Certainly, since the hydrostatic pressure is proportional to the water density, this is completely equivalent to apply the load factor to that water density resulting in a "fictitious" water density (see Fig. 7).

This is not the most common scenario adopted in dam design, but it is considered more acceptable from the structural failure point of view. Eventually, the resulting pressure for every load factor could be translated into an equivalent height of the water above the dam crest (increasing overflow strategy).

5.2.1.3. Material properties. The adopted (average) mechanical properties are the following: both materials, rock and concrete, have been modelled by an isotropic continuum damage model [12]. The analysis assumes uncertainty for the concrete modelling and deterministic for the rock foundation. Consequently, the results evaluate the "structural collapse" rather than the "structure-foundation collapse". The rock foundation is modelled providing appropriate boundary conditions. The rock and concrete mechanical parameters are summarized in Tables 4 and 5, respectively. In Table 5 mean values and standard deviations for the four random variables considered are shown.

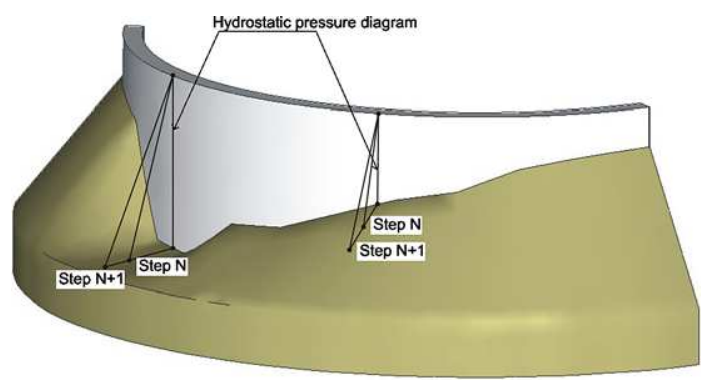

Fig. 7. Load factor evolution.

Table 4

Rock mechanical parameters

Rock foundation

Elastic modulus (MPa)

Poisson's ratio 
Table 5

Concrete mechanical parameters

\begin{tabular}{lll}
\hline & Mean value & Standard deviation \\
\hline Concrete & & 876.8 \\
Elastic modulus $(\mathrm{MPa})$ & 20000 & 0.012 \\
Poisson's ratio & 0.20 & 0.16 \\
Compressive strength $(\mathrm{MPa})$ & 10.0 & 4.5 \\
Tensile strength $(\mathrm{MPa})$ & 1.0 & \\
Fracture energy $(\mathrm{N} / \mathrm{m})$ & 100 & \\
Mass density $\left(\mathrm{Kg} / \mathrm{m}^{3}\right)$ & 2300 & \\
\hline
\end{tabular}

5.2.1.4. Summary of introduced hypotheses. The principal hypotheses considered to solve all the proposed cases are the following:

- Two materials are modelled: rock and concrete.

- No interface elements between the rock foundation and the concrete are considered.

- The rock has been assumed as deterministic.

- The four concrete material properties (Young's modulus, Poisson's ratio, tensile strength and fracture energy) are defined taking into account variations via a probability density function. Their mean values are displayed in Table 5.

- A unique numerical scenario has been adopted. Only the mechanical parameters for concrete are modified.

- Linear geometric analyses are performed.

- Hydraulic fracture phenomena have been included.

- A multi-crack procedure, allowing for multiples failure surfaces, has been considered in all cases.

\subsubsection{Probability of failure}

Numerical results have been obtained, in terms of the load factor, for 100 sets of basic random material properties. Fig. 8 shows the load factor evolution as a function of the horizontal displacement for a given set of input parameters ( $x$-direction of the $P$-node located in the dam crest, see Fig. 6a). The peak load (peak load factor) identifies the maximum carrying capacity of the structure (critical load) under increasing loading processes. The post peak response in Fig. 8 corresponds to the post-critical response of the structure, which would not be traced in an actual case of structural collapse under increasing loads, but allows identifying the

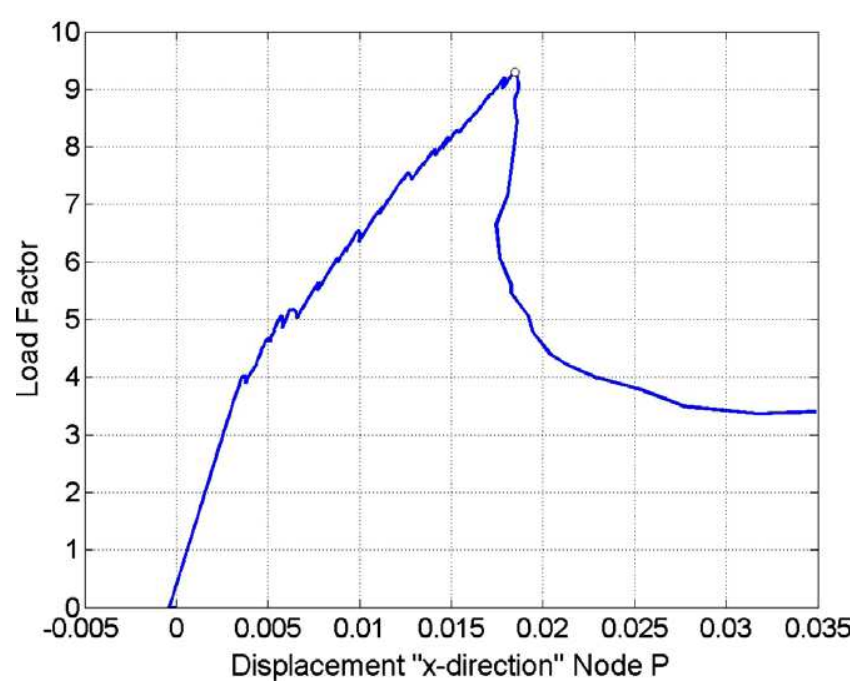

Fig. 8. Dam analysis. Load factor versus $P$-node (see Fig. 6) horizontal displacement. 
failure mode (fracture paths) responsible for the structural collapse. The iso-displacement maps (Fig. 9a) and the geometry in the deformed configuration (Fig. 9b) display the typical failure mechanism. It is formed by the conjunction of two primary macro cracks propagating across the concrete bulk, as shown in Fig. 10a and $\mathrm{b}$. However, the complete dissipative process also considers a number of secondary discontinuity surfaces, in both the dam domain and the rock foundation.

From the 100 CDSA analyses performed with the CSDA, 95 randomly selected are chosen to give the pairs (inputs-outputs) for the NN training. The remaining five are used to test the accuracy of the NN predictions with respect to the "exact" results obtained from the CSDA. Table 6 presents the results of this comparison, while in Fig. 11 the performance of the trained NN for the 95 training patterns is shown. It can be observed that the relative error of peak load factor predicted with the NN and the CDSA is approximately $2.78 \%$ which is considered as adequate for the needs of the subsequent reliability analysis. Once an acceptable trained NN is obtained in predicting the peak load factors, the probability of failure for each test case is estimated by means of the NN-based Monte Carlo Simulation described earlier. Results of this calculation are presented in Fig. 12. The fictitious water density for this calculation takes the deterministic value of $13 \mathrm{KN} / \mathrm{m}^{2}$ which corresponds to an extreme flood condition ( $\sim 8 \mathrm{~m}$ above the maximum water level). From this figure it can be observed that the MCS converges after $10^{8}$ simulations to a probability of failure $p_{\mathrm{f}}=5 \times 10^{-7} \%$.

The feasibility of the proposed methodology for computing the probability of failure in large and complex structural systems is demonstrated in Table 7. In this table, a comparison of the required computing time for developing the three fragility curves of Fig. 13 using the NN based MCS and the basic brute force MCS is presented. The total time required by the proposed NN-MCS can be decomposed in three parts. In the first part is the time required for developing the training and testing sets, running 100 CDSA analyses. The second
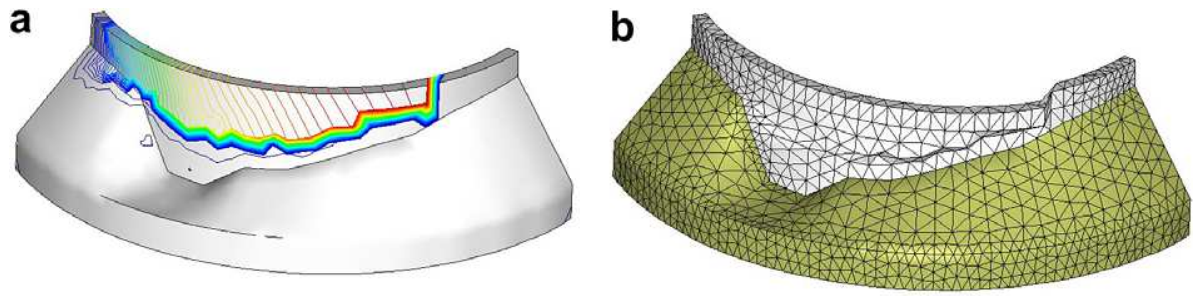

Fig. 9. Dam analysis. (a) Iso-displacement maps. (b) Deformed configuration.
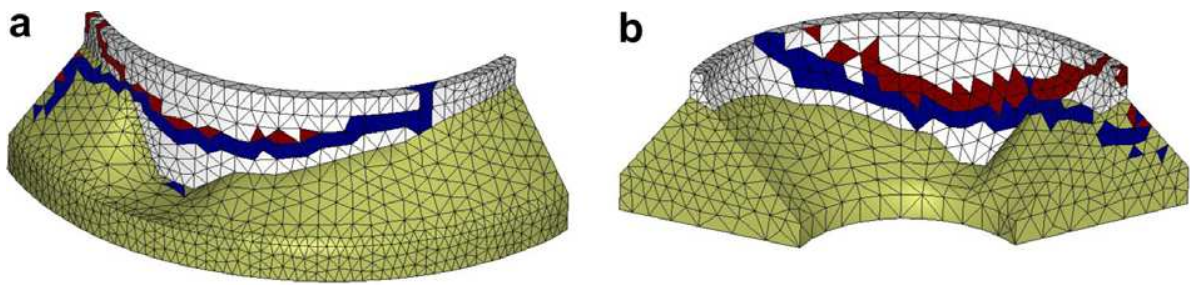

Fig. 10. Dam analysis. Paths of primary cracks, (a) upstream and (b) downstream view.

Table 6

Comparison of CDSA ('exact') and NN predictions of the peak load for five randomly selected input sets of random variables

\begin{tabular}{|c|c|c|c|c|c|c|}
\hline \multirow[t]{2}{*}{ Young's modulus (MPa) } & \multirow[t]{2}{*}{ Poission's ratio } & \multirow[t]{2}{*}{ Tensile strength $(\mathrm{MPa})$} & \multirow[t]{2}{*}{ Fracture energy $(\mathrm{N} / \mathrm{m})$} & \multicolumn{3}{|c|}{ Critical load factor (peak load) } \\
\hline & & & & NN-prediction & CSDA & Error $(\%)$ \\
\hline 20084 & 0.20072 & 0.99553 & 93.100 & 7.50 & 7.29 & 2.78 \\
\hline 19453 & 0.19213 & 0.90258 & 96.994 & 7.55 & 7.45 & 1.38 \\
\hline 18884 & 0.18971 & 0.79474 & 100.570 & 7.19 & 7.30 & 1.44 \\
\hline 21199 & 0.21998 & 1.2994 & 101.590 & 8.14 & 7.94 & 2.52 \\
\hline 20661 & 0.20439 & 1.0997 & 109.960 & 6.80 & 6.97 & 2.42 \\
\hline
\end{tabular}




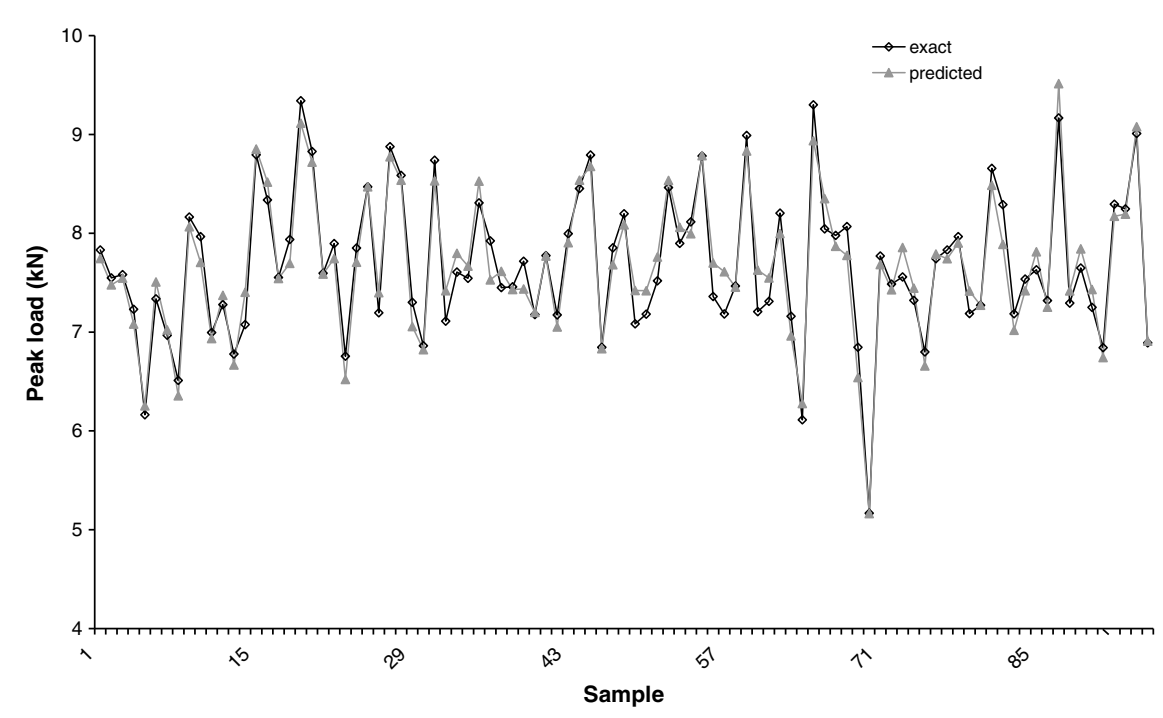

Fig. 11. Performance of the trained NN for the 95 training patterns.

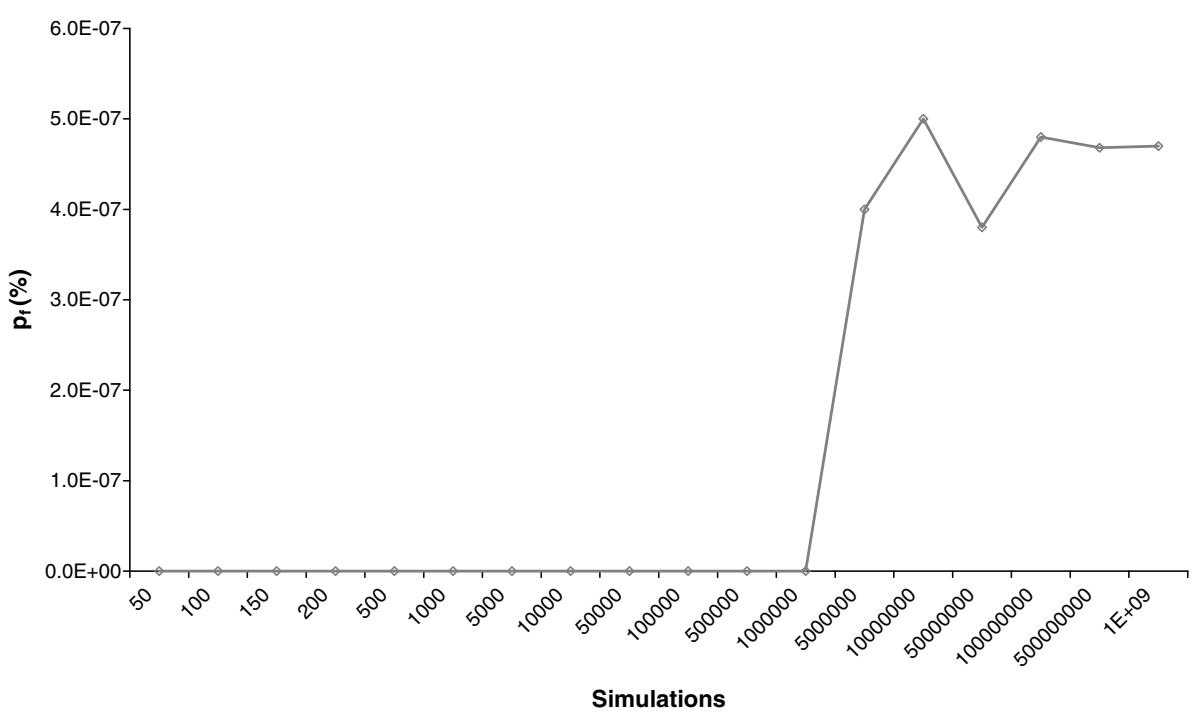

Fig. 12. Probability of failure calculated with the NN based MCS for various numbers of simulations.

Table 7

Comparison of required computing time for developing the three fragility curves of Fig. 13 using the NN based MCS and a brute force MCS

\begin{tabular}{llllll}
\hline & Training/testing set $(\mathrm{h})$ & Training $(\mathrm{h})$ & Prediction $(\mathrm{h})$ & & \multicolumn{2}{c}{ Total } & \\
\cline { 3 - 6 } & & & & Sequential (h) & Sequential (years) \\
\hline NN-MCS & $1.44 \mathrm{E}+03$ & $2.64 \mathrm{E}-03$ & $2.15 \mathrm{E}+00$ & $1.44 \mathrm{E}+03$ & $1.67 \mathrm{E}-01$ \\
Brute force MCS & - & - & - & $1.44 \mathrm{E}+10^{\mathrm{a}}$ & $1.67 \mathrm{E}+06^{\mathrm{a}}$ \\
\hline
\end{tabular}

\footnotetext{
${ }^{\text {a }}$ Estimated.
}

part is the time required for training the $\mathrm{NN}$ while the last one is the simulation time for each water density level $\gamma$. As it can be seen the main part in the total time of the NN-MCS procedure is the first part required for developing the training and testing sets. It can be seen that the enormous computational cost required for a 


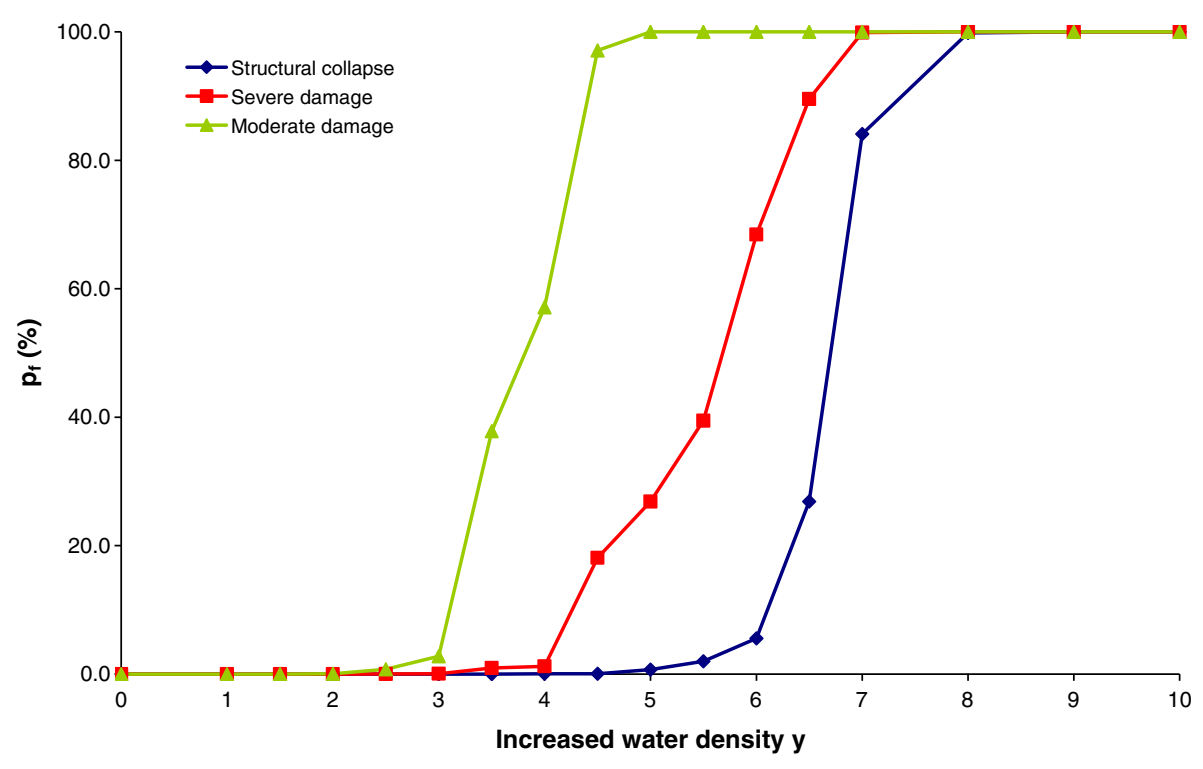

Fig. 13. Flood fragility curves for Scalere dam computed with the NN-MCS approach.

brute-force MCS procedure makes this method prohibitive for this kind of problems. Even if 1000 processors were available the required computing time for such calculation would be $\sim 1670$ years. With the use of NN in the context of a MCS this time is reduced to $\sim 1.500 \mathrm{~h}$. In the present application, a sequential solution procedure was adopted in two personal computers, implementing only the proposed NN-MCS procedure. The total time required for the above calculations was approximately 2 months.

\subsubsection{Fragility curves}

Once the NN based MCS is established for obtaining close predictions of the probability of failure of a dam for a given level of external loading, rigorous calculations of fragility curves can be subsequently obtained by performing a number of reliability analyses associated with various predefined damage levels of the dam and various flood scenarios. These calculations are performed with a minimum additional computational cost. Results of the aforementioned calculation are shown in Fig. 13 where the computed flood fragility curves for Scalere dam are presented. In this figure, each water fictitious density $\gamma$ is assumed to correspond to different water level and therefore to a different flood case scenario. In this work for each fictitious water density $\gamma 1.0 \mathrm{E}+09 \mathrm{NN}$ based MC simulations are performed. In every simulation a check was performed in order to fulfil that the sample drawn was within the bounds of the NN sampling: mean value $\pm 6 \sigma$. In case that a sample was outside of these bounds it was rejected.

Three different damage levels are considered:

- Moderate damage.

- Severe damage.

- Structural collapse.

Moderate damage is assumed to correspond to $60 \%$ of the peak load of the dam and is associated with damages that can be easily repaired without affecting the dam operation. Severe damage is assumed to correspond to the $80 \%$ of the peak load and is associated with extensive damages requiring immediate retrofit and rehabilitation actions. Structural collapse is assumed to correspond to the $100 \%$ of the peak load. In order to obtain the three fragility curves shown in Fig. 13, for each NN based MC simulation the predicted peak load is compared with the limit states for the three damage levels considered. From the fragility curves of Fig. 13, useful conclusions can be derived for the dam behaviour under ordinary and extraordinary loading conditions. 


\section{Concluding remarks}

This paper presents an application of Neural Networks to the reliability analysis of large concrete dams in which failure is predicted with the Continuum Strong Discontinuity Approach. The approximate concepts that are inherent in reliability analysis and the time consuming requirements of repeated structural analyses involved in Monte Carlo Simulation motivated the use of Neural Networks.

The computational effort involved in the conventional Monte Carlo Simulation becomes excessive in largescale problems because of the large sample size and the computing time required for each Monte Carlo run. The use of Neural Networks can practically eliminate any limitation on the scale of the problem and the sample size used for Monte Carlo Simulation provided that the predicted critical load factors, corresponding to different simulations, fall within acceptable tolerances. A Back Propagation Neural Network algorithm is successfully applied to produce approximate estimates of the critical load factors, regardless the size or the complexity of the problem, leading to very close predictions of the probability of failure. The methodology presented could therefore be implemented for predicting accurately and at a fraction of computing time the probability of failure of large and complex structures.

\section{Acknowledgements}

This research was funded by EC in the framework of the Integrity Assessment of Large Concrete Dams European Research Network NW-IALAD. This support is gratefully acknowledged.

\section{References}

[1] Shinozuka M. Basic analysis of structural safety. J Struct Eng ASCE 1983;109:721-39.

[2] Madsen HO, Krenk S, Lind NC. Methods of structural safety. Englewood Cliffs, New Jersey: Prentice-Hall; 1986.

[3] Chrinstensen PT. Reliability and Optimisation of Structural Systems '88. In: Proc of the 2nd IFIP WG7.5. London: Springer-Verlag; 1988.

[4] Oliver J. Modelling strong discontinuities in solids mechanics via strain softening constitutive equations. Part 1: Fundamentals. Int J Numer Meth Eng 1996;39(21):3575-600.

[5] Kam TY, Corotis RB, Rossow EC. Reliability of non-linear framed structures. J Struct Eng ASCE 1983;109:1585-601.

[6] Fujimoto Y, Iwata M, Zheng Y. Fitting-Adaptive Importance Sampling Reliability Analysis. In: Spanos PD, Brebbia CA, editors. Computational Stochastic Mechanics. Computational Mechanics Publications; 1991. p. 15-26.

[7] Pulido JE, Jacobs TL, Prates De Lima EC. Structural reliability using Monte-Carlo simulation with variance reduction techniques on elastic-plastic structures. Comput Struct 1992;43:419-30.

[8] Oliver J. Modelling strong discontinuities in solids mechanics via strain softening constitutive equations. Part 2: Numerical simulation. Int J Numer Meth Eng 1996;39(21):3601-23.

[9] Papadrakakis M, Papadopoulos V, Lagaros ND. Structural reliability analysis of elastoplastic structures using neural networks and Monte Carlo simulation. Comput Methods Appl Mech Eng 1996;136:145-63.

[10] Hurtado JE, Alvarez DA. Neural network based reliability analysis: a comparative study. Comput Methods Appl Mech Eng 2001;191(1-2):113-32.

[11] Adeli H. Neural networks in civil engineering: 1989-2000. Computer-Aided Civil Infrastruct Eng 2001;16(2):126-42.

[12] Oliver J, Huespe AE, Pulido MDG, Chaves E. From continuum mechanics to fracture mechanics: the strong discontinuity approach. Eng Fract Mech 2002;69:113-36.

[13] Oliver J, Huespe AE. Theoretical and computational issues in modelling material failure in strong discontinuity scenarios. Comput Methods Appl Mech Eng 2004;193:2987-3014.

[14] Oliver J, Huespe AE. Continuum approach to material failure in strong discontinuity settings. Comput Methods Appl Mech Eng 2004;193:3195-220.

[15] Oliver J, Huespe AE, Samaniego E, Chaves EWV. Continuum approach to the numerical simulation of material failure in concrete. Int J Numer Anal Methods Geomech 2004;28:609-32.

[16] Oliver J, Huespe AE, Blanco S, Linero DL. Stability and robustness issues in 1 modelling of material failure in the strong discontinuity approach. Comput Methods Appl Mech Eng 2006;195(52):7093-114.

[17] Arrea M, Ingraffea AR. Mixed-mode crack propagation in mortar and concrete. Technical Report No. 81-134, Department of Structural Engineering. New York, Cornell University, 1982.

[18] Frangopol DM, Moses F. Reliability-based structural optimization. In: Adeli H, editor. Advances in design optimization. New York: Chapman-Hall; 1994. p. 492-570.

[19] Schiffmann W, Joost M, Werner R. Optimization of the back-propagation algorithm for training multi-layer perceptrons. Technical Report, University of Koblenz, Institute of Physics, 1993. 
[20] Lagaros ND, Papadrakakis M. Learning improvement of neural networks used in structural optimization. Adv Eng Software 2004;35:9-25.

[21] Fahlman S. An empirical study of learning speed in back-propagation networks. Carnegie Mellon: CMU-CS-88-162; 1988.

[22] Riedmiller M, Braun H. A direct adaptive method for faster back-propagation learning: The RPROP algorithm. In: Ruspini H. (editors), Proc of the IEEE Int Conf on Neural Networks (ICNN), San Francisco, 1993, 586-91.

[23] Riedmiller M. Advanced Supervised Learning in Multi-layer Perceptrons: From Back-propogation to Adaptive Learning Algorithms. University of Karlsruhe, W-76128 Karlsruhe, 1994.

[24] Lagaros ND, Stefanou G, Papadrakakis M. Soft computing hybrid simulation of highly skewed non-gaussian stochastic fields. Comput Methods Appl Mech Eng 2005;194(45-47):4824-44.

[25] Giuseppetti G, Mazzí G, Meghella M, Fanelli M. Evaluation of ultimate strength of gravity dams with curved shape against sliding. Seventh benchmark workshop on numerical analysis dams, September 24-26, 2003, Bucharest, Romania. Available from: http:// www.rocold.ro/themea.htm.

[26] Thematic network on the integrity assessment of large concrete dams (NW-IALAD). Available from: http://nw-ialad.uibk.ac.at/. 